Disponível em:

http://editora.unoesc.edu.br/index.php/race

Race, Joaçaba, v. 14, n. 2, p. 825-842, maio/ago. 2015

\title{
ANÁLISE DOS IMPACTOS GERADOS PELO PROGRAMA MINHA CASA MINHA VIDA NO ÍNDICE DE VELOCIDADE DE VENDAS NA REGIÃO METROPOLITANA DO RECIFE
}

\section{Analysis of impacts generated by The Minha Casa Minha Vida Program in Sales Speed Index in Metropolitan Region of Recife}

Sylvia Karla Gomes Barbosa

E-mail: sylvia.economia@gmail.com

Mestre em Administração e Desenvolvimento Rural com ênfase em Políticas Públicas e Desenvolvimento Rural Sustentável pela Universidade Federal Rural de

Pernambuco; Graduada em Economia pela Universidade Federal Rural de Pernambuco; Professora dos Cursos de Processos Gerenciais e Marketing da Faculdade de Tecnologia, Gestão e Marketing.

José Ferreira Irmão

E-mail: jferreirairmao@gmail.com

Doutor em Economia pela University College London, Inglaterra; Mestre em Economia pela Yale University, Estados Unidos; Professor do Programa de Pósgraduação em Administração e Desenvolvimento Rural pela Universidade Federal Rural de Pernambuco. Endereço para contato: Rua Dom Manuel de Medeiros, s/n - Dois Irmãos, 52171900, Recife, Pernambuco, Brasil.

André de Souza Melo

E-mail: andredesouzam@gmail.com

Doutor e Mestre em Economia pela Universidade Federal de Pernambuco; Professor do Programa de Pós-Graduação em Administração e Desenvolvimento Rural da Universidade Federal Rural de Pernambuco.

Artigo recebido em 16 de março de 2015. Aceito em 30 de abril de 2015. 


\title{
Resumo
}

O presente trabalho teve como principal objetivo analisar os impactos gerados pelo Programa Minha Casa Minha Vida (PMCMV) no Índice de Velocidade de Vendas da Região Metropolitana do Recife (RMR) entre 2002 e 2013. O mercado imobiliário do Grande Recife obteve avanços significativos, principalmente entre 2004 e 2009, quando os financiamentos habitacionais praticamente triplicaram. Por essa razão, surgiu o interesse em estudar esse mercado diante da perspectiva da política pública, partindo do pressuposto de que essa política é uma ferramenta de interferência positiva nesse mercado. Assim, analisaram-se as políticas habitacionais do Governo Fedeal, com ênfase no PMCMV, e seus impactos na redução do déficit habitacional na RMR. Aplicou-se, finalmente, o modelo econométrico de intervenção para verificar, de forma mais profunda, os efeitos dessa política habitacional sobre a demanda e a oferta de imóveis na região. Palavras-chave: Programa Minha Casa minha Vida. Índice de Velocidade de Vendas. Região Metropolitana do Recife.

\section{Analysis of impacts generated by The Minha Casa Minha Vida Program in Sales Speed Index in Metropolitan Region of Recife}

\begin{abstract}
This study had, as main purpose, to analyze the impacts of the Federal Government Program Minha Casa Minha Vida (PMCMV) on the Sales Speed Index in the Metropolitan Region of Recife (RMR) during 2002 and 2013. The real estate market of Greater Recife has made significant progress, especially between 2004 and 2009, when the housing loans nearly tripled. This was the reason for studying this real estate market and the Government policy that stimulated its development in the period. Following this, housing policies of the Federal Government were analyzed, with special emphasis to the PMCMV policy, and its impact on reducing the housing deficit on the RMR. It was applied, finally, the econometric model of intervention to check, on a deeper level, the effects of housing policy on the demand and supply of real estate in the region. Keywords: Minha Casa Minha Vida Program. Sales Speed Index. Metropolitan Region of Recife (RMR).

\section{INTRODUÇÃO}

O setor da construção civil tem se destacado bastante nos últimos anos, principalmente no que se refere à geração de renda e de empregos. De acordo com o Instituto Brasileiro de Geografia e Estatística (2013), o PIB da construção civil obteve um crescimento de 3,8\% no segundo trimestre de 2013 em relação ao primeiro trimestre do mesmo ano, sendo o segundo setor que mais cresceu nesse período, ficando atrás apenas do setor agropecuário. Quanto ao número de empregos, a Pesquisa Anual da Indústria da Construção revelou que cerca de 2,7 milhões de pessoas trabalhavam nesse setor, ou seja, 200 mil novos empregos foram gerados quando comparado ao ano 2010 (IBGE, 2011). Ainda, de acordo com essa pesquisa,
\end{abstract}


as obras de infraestrutura absorvem $41,4 \%$ do total da receita bruta das empresas e, em segundo lugar, a construção de prédios, com 39,5\%.

Em relação à Região Metropolitana do Recife (RMR), também é possível constatar a grande relevância do setor da construção civil para a região, tanto na questão econômica quanto na social. A Pesquisa Mensal de Emprego revelou que, em setembro de 2013, 8,6\% da população ocupada da RMR pertenciam ao setor da construção civil, sendo esse percentual superior ao dos dois últimos anos (IBGE, 2013).

No entanto, a RMR carrega consigo grandes problemas sociais, sendo um deles o déficit habitacional, ${ }^{1}$ que chega a ser aproximadamente $50 \%$ do total do déficit habitacional do Estado de Pernambuco (FUNDAÇÃO JOÃO PINHEIRO, 2013).

Assim, o Governo Federal, na tentativa de mudar esse cenário e melhorar a situação, não apenas da população da RMR, mas de todo o Brasil, implantou o Programa Minha Casa Minha Vida (PMCMV), que tem como principal objetivo facilitar a aquisição de moradias para toda a população, em especial a de baixa renda.

Dessa forma, o objetivo central deste trabalho consistiu em analisar os impactos do Programa Minha Casa Minha Vida no Índice de Velocidade de Vendas $(I V V)^{2}$ da Região Metropolitana do Recife durante o período de 2002 a 2013, partindo do pressuposto de que o PMCMV impactou de maneira positiva o IVV, ou seja, a partir da implantação do Programa, em 2009, a velocidade das vendas de imóveis residenciais cresceu.

\section{ASPECTOS COMPLEMENTARES}

Ao longo dos anos, o mercado imobiliário da RMR tem presenciado um crescimento bastante acelerado, principalmente após a criação do programa habitacional do Governo Federal no ano 2009, o Programa Minha Casa Minha Vida. Pensando nisso, as subseções a seguir discutem a política habitacional brasileira ao longo dos anos, assim como delineiam o PMCMV e, por último, descrevem o cenário do mercado imobiliário da RMR antes e depois do Programa.

\section{1 POLÍTICA HABITACIONAL DO BRASIL: UM BREVE HISTÓRICO}

O ponto de partida da intervenção do Governo Federal no setor habitacional ocorreu a partir dos anos da década de 1930, por meio da criação dos Institutos de Aposentadorias e Pensões (IAPs), sendo permitida pela legislação a utilização 
de parte da receita dos caixas para a construção de casas. No entanto, somente na década de 1940, com a junção dos IAPs, foi criada a Fundação da Casa Popular, que era inteiramente voltada para a construção de moradias de interesse social. Porém, em razão da falta de recursos e de insumos, da elevação do preço da terra, entre muitas outras dificuldades, a Fundação da Casa Popular nos anos 1950 enfraqueceu e o Sistema Financeiro de Habitação (SFH) ganhou espaço em 1964, depois do Golpe Militar. As operações do SFH eram realizadas pelo Banco Nacional de Habitação (BNH), por meio do Fundo de Garantia por Tempo de Serviço (FGTS) e do Sistema Brasileiro de Poupança e Empréstimos (SBPE), entretanto, a principal característica que marcava a economia brasileira, nessa época, era sua instabilidade, e, assim, retiradas abruptas ocorriam tanto do FGTS quanto do SBPE, e, além disso, a inadimplência se instaurou no setor habitacional, levando, dessa forma, o Sistema Financeiro de Habitação ao declínio em 1986, sendo então incorporado à Caixa Econômica Federal (CARDOSO et al., 2013).

Ainda dentro deste contexto, a Caixa Econômica Federal (2011) afirma que, de 1986 a 2002, apenas a iniciativa privada assumiu o controle do setor habitacional, direcionando-o para a população que se mantinha em um patamar de renda mais elevado. E, somente, em 2003, o cenário começa a mudar, sendo então criado o Ministério das Cidades e a Política Nacional de Habitação (PNH), em 2004. Não obstante, em 2007, nasce o Programa de Aceleração do Crescimento (PAC), com a finalidade de promover o desenvolvimento para todas as regiões brasileiras, e parte dos investimentos desse Programa era direcionada ao setor habitacional do país; inclusive, em 2009, 83,9\% dos investimentos de $\mathrm{R} \$ 41,8$ bilhões do PAC foram para o setor em discussão.

Porém, em razão da crise internacional que se instaurou a partir de 2008, o Governo Federal precisou implantar outras medidas que estimulassem o crescimento econômico da nação, e, dentro dessa perspectiva, o setor da construção civil foi o mais beneficiado, considerando seu retorno socioeconômico elevado, favorecendo, assim, o surgimento do Programa Minha Casa Minha Vida (CAIXA ECONÔMICA FEDERAL, 2011).

\section{2 PROGRAMA MINHA CASA MINHA VIDA (PMCMV)}

De acordo com Shimizu (2010), o Programa Minha Casa Minha Vida I foi criado a partir da Medida Provisória n. 459, em 25 de março de 2009, sendo 
regulamentado pelo Decreto n. 6.819, em abril de 2009, e somente, em 07 de julho de 2009 transformado na Lei n. 11.977.

Como se sabe, a primeira fase do PMCMV já foi concluída, e atualmente esse Programa se encontra na execução da sua segunda fase. Não obstante, a primeira fase do Programa estimou um investimento de $\mathrm{R} \$ 34$ bilhões, sendo esse recurso proveniente da União, do FGTS e do BNDES. Na segunda fase, o Programa se apresentou mais ousado, considerando que a primeira se mostrou satisfatória, pelo menos no cumprimento das metas estabelecidas, e propôs um investimento de R\$ 71,7 bilhões, em uma perspectiva de ser construído o dobro de moradias da primeira fase (de um para dois milhões de unidades habitacionais). Vale ainda ressaltar que o Programa busca beneficiar a população que se mantém no nível de renda de zero a dez salários mínimos, sendo as famílias que recebem de zero a três salários mínimos beneficiadas com a isenção total do seguro, as de três a seis, beneficiadas parcialmente no financiamento, além da redução dos custos do seguro e do acesso ao FGTS e, por fim, as de seis a dez, com apenas uma redução nos custos do seguro e acesso também ao FGTS (SHIMIZU, 2010).

No que se refere ao valor do financiamento, a Tabela 1 apresenta, de maneira bem detalhada, a situação nos Programas PMCMV1 e PMCMV2.

Tabela 1 - Financiamento por faixa de renda do PMCMV

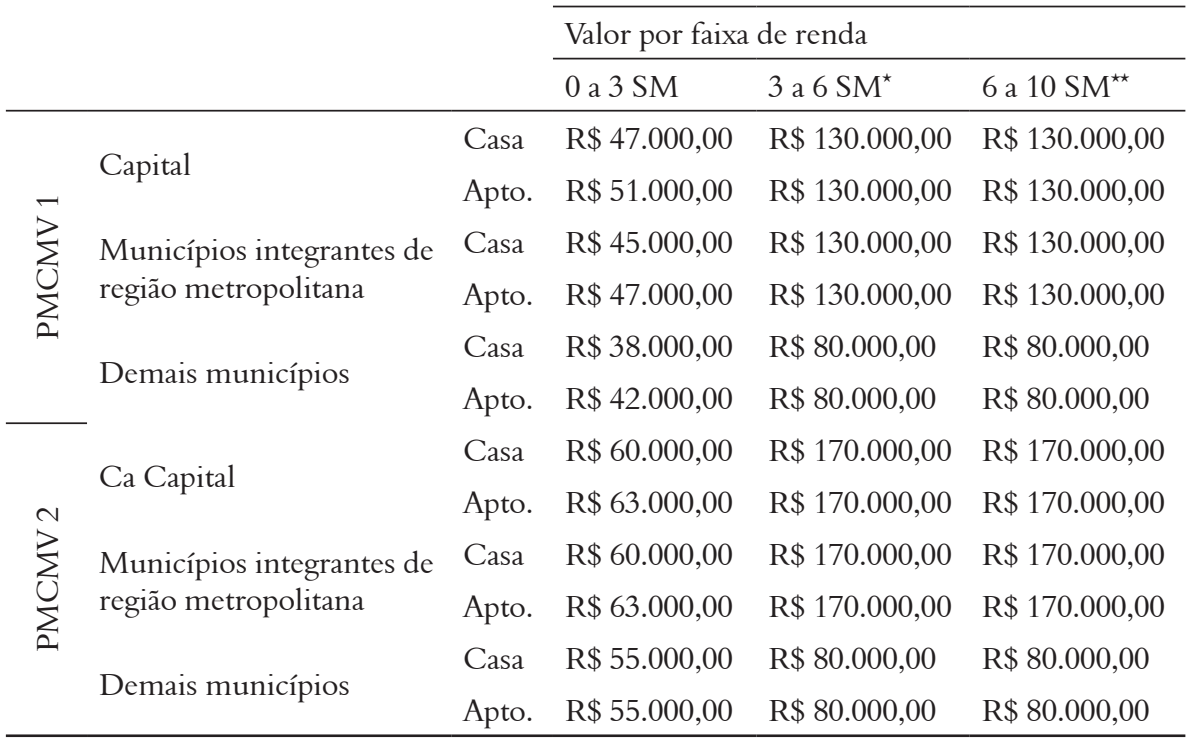

Fonte: Cardoso e Aragão (2012).

Notas: ${ }^{\star}$ Taxa de juros nominal de $6 \%$ a.a.

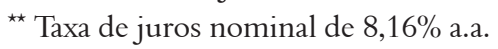


Tendo tido uma perspectiva geral do Programa Minha Casa Minha Vida dentro do âmbito nacional, vale enfatizar sua atuação na Região Metropolitana do Recife, a qual é o objeto de estudo deste trabalho. Assim, segundo Becher (2013), o mercado imobiliário da RMR mantém-se aquecido por dois motivos: primeiramente pela disponibilidade de terrenos e depois pelo PMCMV. Ainda complementa que o Índice de Velocidade de Vendas (IVV), um indicador de grande importância para o setor habitacional da RMR, vem se apresentando bastante elevado, principalmente pelos imóveis que contemplam o PMCMV em seu mecanismo de financiamento.

De acordo com o noticiário:

\begin{abstract}
A influência do Minha Casa é tanta que a Fiepe resolveu divulgar separadamente os indicadores das vendas dos imóveis financiados pelo Programa e os que não são financiados por ele. Isso porque, bairros onde se constatou presença marcante de vendas, através desse financiamento habitacional, estavam elevando bruscamente a média geral do IVV. No final do ano passado, por exemplo, o índice considerando as vendas por meio desse Programa era de $16,4 \%$, enquanto o IVV que desconsiderava as vendas do Minha Casa Minha Vida era de 8\%. (ÂNGELA, 2013).
\end{abstract}

É possível perceber a representatividade agressiva do Programa Minha Casa Minha Vida na RMR, cerca de metade do IVV é composto por imóveis que faz uso desse Programa habitacional, assim, na próxima subseção, será melhor analisado o cenário da RMR, tomando por base o PMCMV, antes e depois de sua implantação.

\title{
2. 3 MERCADO IMOBILIÁRIO DA RMR: ANTES E DEPOIS DO PMCMV
}

O Índice de Velocidade de Vendas é considerado um importante indicador para o setor habitacional. Ele é obtido a partir do somatório das vendas de imóveis novos da RMR, divididas pelo somatório das ofertas, sendo, então, o resultado referente à velocidade das vendas ocorridas, em determinado mês, frente à oferta de 100 imóveis, ou seja, se o IVV correspondeu a 10 significa que 10 vendas ocorreram para um total de 100 unidades habitacionais ofertadas (ASSOCIAÇÃO DAS EMPRESAS DO MERCADO IMOBILIÁRIO DE PERNAMBUCO, 2013). 
Não obstante, é possível observar, a partir do Gráfico 1, que o mercado imobiliário do Grande Recife pode ser caracterizado em dois momentos. No primeiro, de 2002 a 2007, o mercado habitacional encontrava-se em um nível bastante baixo, mesmo no período em que foi criado o Ministério das Cidades e a Política Nacional de Habitação. E o mais impressionante é que, em 2007, já se percebia um movimento crescente da curva, exatamente na fase de implantação do PAC I. No segundo momento, que se estende de 2008 a 2013, nota-se que o setor habitacional, entre 2008 e 2009, encontrava-se em declínio, em decorrência da crise internacional que atingiu o mundo. Nos anos seguintes, contudo, houve um grande salto no IVV, tendo o PMCMV como principal responsável dessa alavancada; além disso, observa-se que o IVV, nesse momento, permaneceu dentro do intervalo de 10 e 20 , tendo períodos em que atingiu picos que chegaram quase a 30, ressaltando que mesmo estando em um patamar mais elevado, esse indicador ainda permanece bastante oscilatório.

Gráfico 1 - Índice de Velocidade de Vendas na RMR - De 2002 a 2013

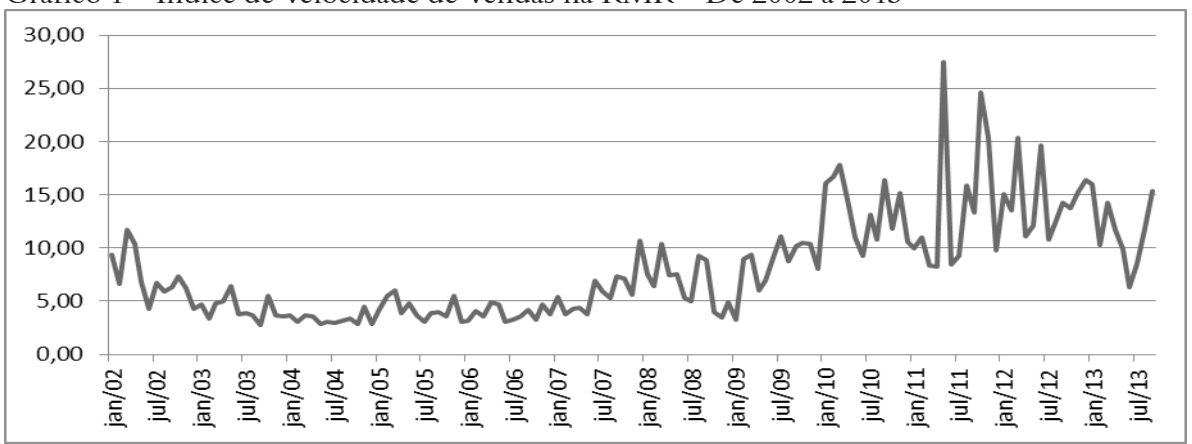

Fonte: adaptado de Associação das Empresas do Mercado Imobiliário de Pernambuco (2013).

Considerando que o IVV é um indicador derivado das ofertas e das vendas dos imóveis novos da Região Metropolitana do Recife, vale desmembrálo, como é possível verificar no Gráfico 2, de maneira que seja possível perceber o comportamento da demanda e da oferta separadamente. Assim, quanto à oferta de imóveis, verifica-se que de 2002 a 2007 o seu comportamento se manteve basicamente estável; a partir de 2008, observa-se um crescimento acelerado, que se estende até 2010. A partir de então, as ofertas de imóveis na Região Metropolitana do Recife iniciam uma desaceleração, inclusive, em 2011, alcançam patamares inferiores aos vistos até então. No entanto, nota-se que esse comportamento não é permanente, pois em 2012 as ofertas voltam a crescer. 
Gráfico 2 - Ofertas versus vendas de imóveis novos na RMR

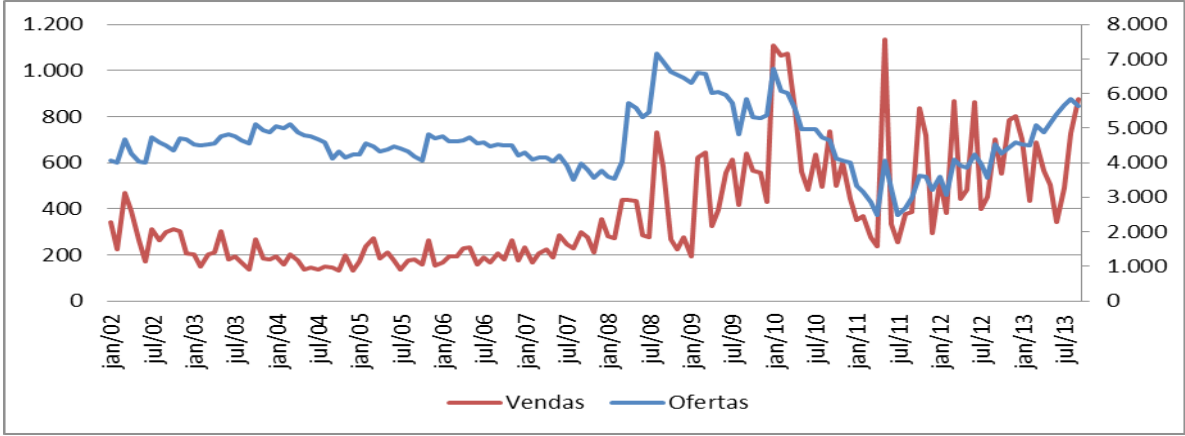

Fonte: adaptado de Associação das Empresas do Mercado Imobiliário de Pernambuco (2013).

Em relação às vendas, é possível verificar que o comportamento dessa série se mostra relativamente semelhante ao que ocorre nas ofertas, principalmente no período que se estende de 2002 a 2007, isto é, um comportamento estável. A partir de 2008, percebe-se que se iniciou um processo de expanção das vendas, porém, esse crescimento ocorreu de forma bastante oscilatória, alcançando em janeiro de 2010 e em julho de 2011 picos impressionantes.

Quanto aos lançamentos de unidades habitacionais, a partir do Gráfico 3, nota-se que, de 2002 a 2007, essa quantidade se manteve entre 0 e 500, ao passo que, entre 2008 e 2013, a quantidade de lançamentos se tornou mais representativa, atingindo picos bastante expressivos.

Gráfico 3 - Lançamento de imóveis residenciais na RMR

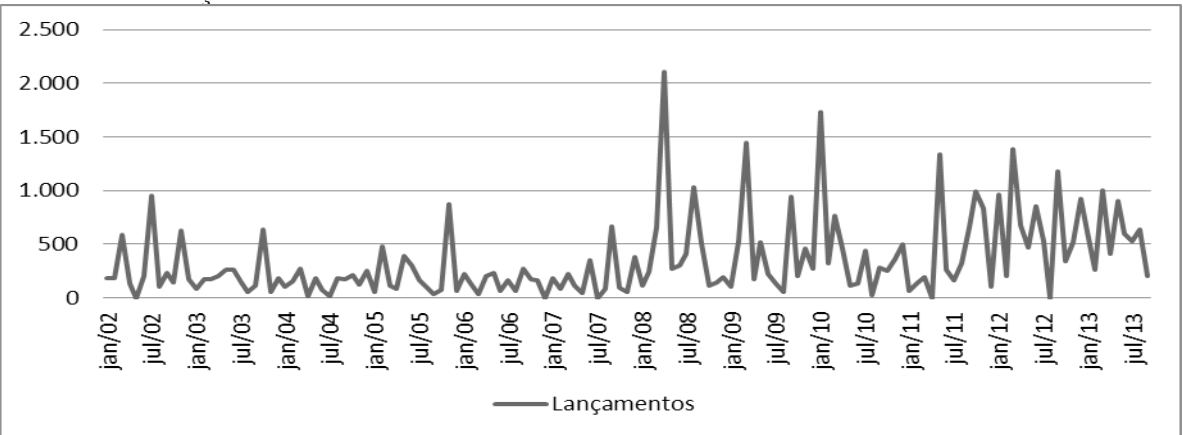

Fonte: adaptado de Associação das Empresas do Mercado Imobiliário de Pernambuco (2013). 
Assim, percebe-se que, em todas as variáveis discutidas, o PMCMV interfere de maneira a alavancar esses indicadores, mesmo que seja de forma irregular, isto é, oscilatória. Essa análise, no entanto, é feita a partir da visão dos gráficos representados, sendo necessária para a confirmação dessas análises a utilização de um modelo mais sofisticado, que possa incrementar essas discussões. Dessa forma, a fim de agregar as prévias conclusões observadas, este artigo se propôs a utilizar um modelo econométrico conhecido como Modelo de Função Intervenção, que será explicado na seção seguinte.

\section{ASPECTOS METODOLÓGICOS}

\section{1 DADOS}

Neste trabalho, foram utilizadas observações mensais acerca do Índice de Velocidade de Vendas (IVV) dos imóveis novos na Região Metropolitana do Recife para o período que se estende de janeiro de 2002 a setembro de 2013, sendo esses dados retirados da pesquisa realizada pela Federação das Indústrias do Estado de Pernambuco.

No que se refere à representação do Programa Minha Casa Minha Vida, foi construída uma variável binária que assume valor 0 para o espaço de tempo em que o Programa não existia (de janeiro de 2002 a fevereiro de 2009) e 1 para o período em que ele passa a vigorar (de março de 2009 a outubro de 2013).

\section{2 MODELO ECONOMÉTRICO}

Normalmente, ao se estudar o impacto de uma política pública em uma determinada série temporal, utiliza-se uma ferramenta econométrica conhecida por Função de Intervenção. Nesse tipo de modelo, tem-se primeiramente uma variável dependente, que, por meio de sua trajetória ao longo do tempo, permitirá analisar se foi impactada ou não por uma variável independente; esta, por sua vez, é expressa por uma variável do tipo binária, que assume valores de 0 e 1 . Desse modo, por meio do modelo proposto por Box, Jenkins e Reinsel (1994), a Função de Intervenção é expressa da seguinte maneira: 


$$
Y_{t}=d_{1} Y_{t-1}+1 / 4+d_{r} Y_{t-r}+w_{0} I_{t-b}-w_{1} I_{t-b-1}-1 / 4-w_{s} I_{t-b-s}
$$

Sendo $Y_{t}$ expresso mediante a sua memória autorregressiva de ordem $r$, e $I_{t-b}$ por seus valores defasados até a ordem $s$. Os parâmetros d e w representam a relação entre as variáveis independentes e a variável dependente. Em relação ao $b$, este indica o fator de retardamento do impacto de $I_{t}$ em $Y_{t}$, ou seja, se ocorrer de imediato o impacto de $I_{t}$ em $Y_{t}, b$ equivale a zero, se ocorrer depois de um período, $b$ equivale a um e assim por diante.

Colocando os termos da variável dependente para antes da igualdade e os da variável independente para depois, pode-se reescrever a expressão 3.2.1 assim:

$$
Y_{t}-d_{1} Y_{t-1}-1 / 4-d_{r} Y_{t-r}=w_{0} I_{t-b}-w_{1} I_{t-b-1}-1 / 4-w_{s} I_{t-b-s}
$$

Substituindo a memória autorregressiva de $Y_{t}$ e os valores defasados de $X_{t-b}$ por $\boldsymbol{B}^{m}$, que, por sua vez, representa um operador de defasagem, tem-se:

$$
\left(1-d_{1} B-1 / 4-d_{r} B^{r}\right) Y_{t}=\left(w_{0}-w_{1} B-1 / 4-w_{s} B^{s}\right) I_{t-b}
$$

Sendo, então, $Y_{t}$ determinado por $\mathrm{d}(\boldsymbol{B})=\left(1-\mathrm{d}_{1} \boldsymbol{B}-1 / 4-\mathrm{d}_{t} \boldsymbol{B}^{r}\right)$ e $I_{t-b}$ por w $(\boldsymbol{B})=\left(\mathrm{w}_{0}-\mathrm{w}_{1} \boldsymbol{B}-1 / 4-\mathrm{w}_{s} \boldsymbol{B}\right)$. Igualando mais uma vez os dois termos, como feito na expressão 3.2.2 e 3.2.3, tem-se:

$$
d(B) Y_{t}=w(B) I_{t-b} \text { ou } Y_{t}=d(B)^{-1} w(B) I_{t-b}
$$

Logo, d $(\boldsymbol{B})^{-1} \mathrm{~W}(\boldsymbol{B})$ indica a função impulso-resposta de $I_{t-b}$ em $Y_{t}$ E a fim de simplificar ainda mais a análise da Função de Intervenção, pode-se considerar d $(\boldsymbol{B})^{-}$ ${ }^{1} \mathrm{~W}(\boldsymbol{B})=v(\boldsymbol{B})$, e, assim, a expressão é reescrita da seguinte forma:

$$
Y_{t}=v(\boldsymbol{B}) I_{t-b}
$$

Vale ressaltar que de acordo com Mills (1990), a relação entre $Y_{t}$ e $I_{t-b}$ não é determinada pelo tempo, ou seja, não é determinística e, sim, está vinculada a uma distribuição de probabilidade, assim, essa relação é aleatória e deve conter o termo de erro $\left(N_{t}\right)$, que, por sua vez, pode ser representado pelo modelo de ruído do ARMA (ou ARIMA), sendo independentemente distribuído e seu valor esperado é 0 . 


$$
Y_{t}=v(\boldsymbol{B}) I_{t-b}+N_{t}
$$

Sendo $N_{t}=\mathrm{f}^{-1}(\boldsymbol{B}) \mathrm{q}(\boldsymbol{B}) \mathrm{e}_{t}$

Outra questão relevante diz respeito à forma em que a intervenção pode ser observada, em nível, por pulso ou por pulso expandido. Em nível, a intervenção ocorre em determinado período e permanece ao longo do tempo. Por pulso, a intervenção acontece em um período e deixa de intervir, e por pulso expandido, a intervenção persiste por algum tempo e em seguida estaciona (MILLS, 1990).

\section{3 IDENTIFICAÇÃO, ESTIMAÇÃO E DIAGNÓSTICO}

Conforme Mills (1990), o procedimento de identificação, como o próprio nome já indica, é determinado pela busca de estimativas preliminares dos parâmetros $\mathrm{d}, \mathrm{w}, \mathrm{f}$ e q, que pode ser realizada mediante os mínimos quadrados ordinários. Já para as estimativas de $r, s$ e $b$, faz-se uso da análise das características da intervenção, se o impacto ocorre de imediato ou depois de algum período, e se permanece ou somente acontece por pulsos. Por último, a estimação do modelo de ruído é estimada como um ARMA da seguinte forma:

$$
\hat{n}_{t}=y_{t}-\hat{v}(B) I_{t}
$$

Depois das estimativas para a função de intervenção e para o modelo de ruído, o modelo completo pode ser estimado por máxima verossimilhança por meio dos mínimos quadrados não lineares. Dessa maneira:

$$
d(\boldsymbol{B})=\mathrm{d}_{r}(\boldsymbol{B}) \mathrm{f}_{p}(\boldsymbol{B}), c(\boldsymbol{B})=\mathrm{f}_{p}(\boldsymbol{B}) \mathrm{W}_{s}(\boldsymbol{B}) \mathrm{e} b(\boldsymbol{B})=\mathrm{d}_{r}(\boldsymbol{B}) \mathrm{q}_{q}(\boldsymbol{B})
$$

Organizando a expressão 3.3.2, tem-se:

$$
d(\boldsymbol{B}) y_{t}=c(\boldsymbol{B}) x_{t}+b(\boldsymbol{B}) a_{t}
$$

Sendo o erro $\left(a_{t}\right)$ representado da seguinte forma: 


$$
a_{t}=y_{t}+d_{1} y_{t-1}+1 / 4+d_{p+r} y_{t-p-r}+c_{0} x_{t-b}+c_{1} x_{t-b-1}+1 / 4+c_{p-s} x_{t-b-p-s}+b_{l} a_{t-1}+1 / 4+b_{r+q}
$$

Substituindo os parâmetros por b, tem-se:

$$
\mathrm{b}=\left(\mathrm{w}_{0}, \mathrm{w}_{1}, 1 / 4, \mathrm{w}_{\mathrm{s}} ; \mathrm{d}_{1}, 1 / 4, \mathrm{~d}_{\mathrm{r}} ; \mathrm{f}_{1}, 1 / 4, \mathrm{f}_{\mathrm{p}} ; \mathrm{q}_{1}, 1 / 4, \mathrm{q}_{\mathrm{q}}\right)
$$

Assim, é possível obterem-se os estimadores condicionais de máxima verossimilhança ou de mínimos quadrados não lineares, minimizando-os assim:

$$
S=\sum_{t=1}^{n} a_{t}^{2}(\boldsymbol{\beta})
$$

O diagnóstico foi realizado por meio de três procedimentos, o exame de ajuste $\left(\mathrm{R}^{2}\right)$, a significância dos parâmetros (estatística t), e, por fim, verificou-se se os $m$ resíduos são ruído branco. Para tal, utilizou-se a estatística Ljung-Box, sendo calculada para $T$ observações e $k$ defasagens da seguinte forma:

$$
Q=T(T+2) \sum_{k=1}^{K}(T-k)^{-1} r_{k}^{2}
$$

Com distribuição $\chi^{2}$ e com $K-p-q$ graus de liberdade.

Vale ressaltar que, de acordo com Enders (1995), a escolha entre os modelos alternativos ocorreu de acordo com os critérios Akaike Information Criterion (AIC) e Schwartz Bayesian Criterion (SBC).

\section{RESULTADOS E DISCUSSÃO}

Este trabalho se valeu de apenas um modelo de intervenção, sendo este referente ao impacto causado pelo Programa Minha Casa Minha Vida, no Índice de Velocidade de Vendas de imóveis residenciais novos na RMR.

Dessa forma, o primeiro passo foi dado verificando se a série em questão (IVV) era estacionária. Para isso, foi realizado o teste de Dickey-Fuller Aumentado (ADF) e notou-se que a série possuía raiz unitária, contudo, diferenciando-a uma única vez se tornava estacionária. 
No que se refere à identificação dos estimadores, tem-se que o melhor modelo, considerando o $\mathrm{R}^{2}$ ajustado, a estatística $t$ e principalmente o teste de LjungBox, foi o descrito na Tabela 2, na qual é possível observar todos os valores estimados. Vale ressaltar que a estimação foi feita a partir do método de verossimilhança, já descrito na seção 3. 3.

Nesse modelo, o coeficiente ${ }_{w_{0}}$ foi significativo ao nível de $10 \%$, o coeficiente $d_{1}$ também foi significativo, a um nível de significância ainda menor, 1\%, ocorrendo o mesmo com o $f_{1}$. Em relação ao $\mathrm{R}^{2}$ ajustado, termo de determinação múltipla, foi equivalente a 63\%. E as estatísticas Q(8), Q(16) e Q(32) não confirmam a hipótese de ruído branco, ou seja, o termo de erro não é estacionário, podendo ser considerado um problema para o modelo.

Tabela 2 - Estimação do Modelo de Intervenção

\begin{tabular}{ll}
\hline Coeficientes & Intervenção (Oferta X PMCMV) \\
\hline$w_{0}$ & 2,655747395 \\
$w_{1}$ & $(1,511130229) 0,08107350^{\star}$ \\
$d_{1}$ & - \\
$d_{2}$ & $-0,985651661(0,023105474) 0,00000000^{\star}$ \\
$f_{1}$ & - \\
$f_{2}$ & $-0,777838373(0,054610435) 0,00000000^{\star}$ \\
$f_{3}$ & - \\
$\mathrm{R}^{2}$-ajustado & - \\
Q(8) & 0,631146 \\
Q(16) & 17,772 \\
Q $(24)$ & $(0,003247)$ \\
AIC & 32,872 \\
Fon & $(0,001781)$ \\
& 44,477 \\
& $(0,002027)$ \\
& 1008,07627 \\
& \\
&
\end{tabular}

Fonte: os autores.

Nota: ${ }^{\star}$ Indica a significância para a estatística t.

Os valores entre parênteses correspondem ao desvio padrão para os coeficientes estimados e ao nível de significância para a estatística Q. 
Quanto à função de Impulso-Resposta, foi possível observar que o impacto do PMCMV se comporta de maneira oscilatória (Gráfico 4), ocorrendo, então, no primeiro momento, um salto no IVV, enquanto no terceiro, uma queda abrupta. À medida que vai passando o tempo, entretanto, as oscilações são menos acentuadas.

Gráfico 4 - Função Impulso-Resposta

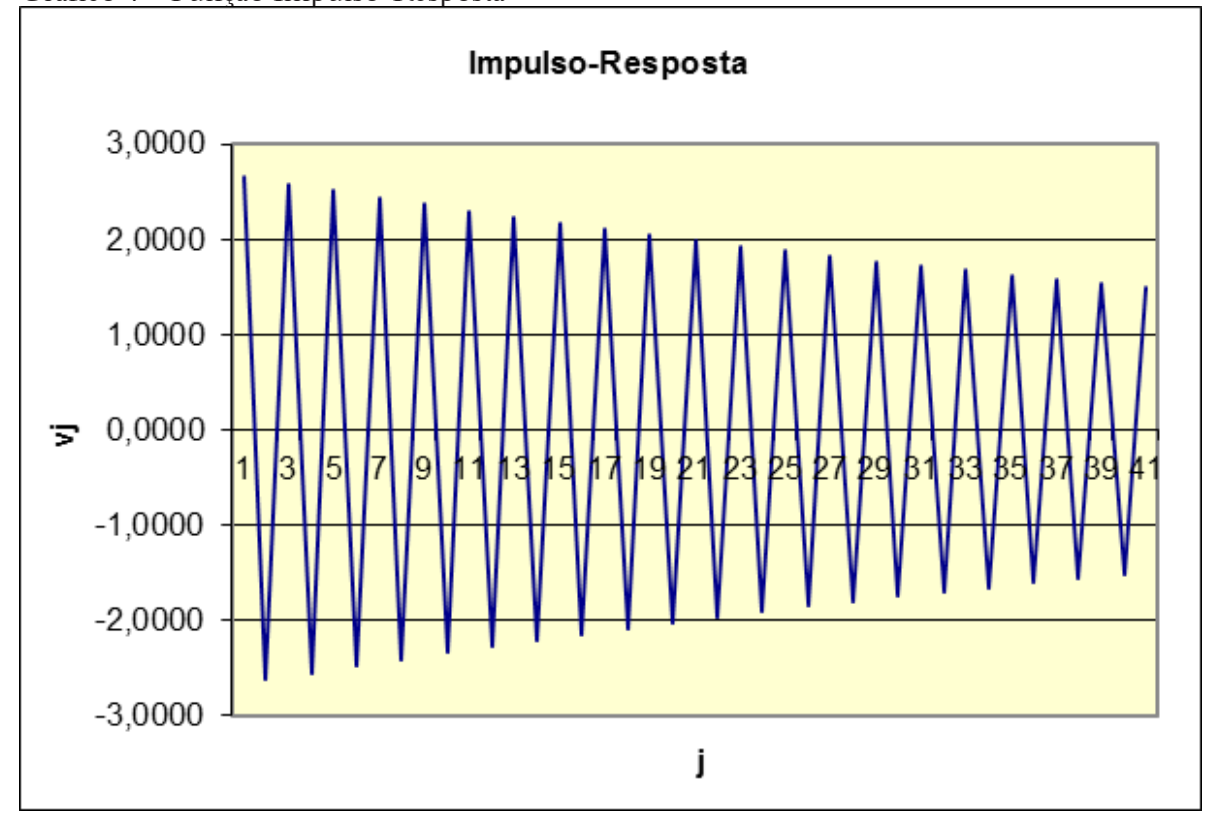

Fonte: os autores.

Logo, mediante o Gráfico 4, fica evidente o comportamento irregular que o PMCMV exerce sobre o IVV.

\section{CONSIDERAÇÕES FINAIS}

Os resultados mostraram que o Programa Minha Casa Minha Vida não impactou apenas de modo positivo o Índice de Velocidade de Vendas, muito pelo contrário, houve momentos em que as vendas realmente atingiram picos bastante significativos, assim como quedas muito relevantes. O que vale ressaltar é que este trabalho considerou apenas o programa habitacional como propulsor das vendas/ IVV. Sabe-se, no entanto, que existem diversas variáveis que podem influenciar esse 
modelo e dar maior robustez às justificativas das quedas e saltos da venda, como renda, taxa de juros, entre outras, mas como o objetivo deste trabalho foi apenas analisar o impacto do PMCMV no IVV e não verificar os fatos que levaram a série a ter tal comportamento, o trabalho se restringiu a dizer que, de fato, houve a influência do PMCMV no IVV, e, quanto ao comportamento, foi um tanto irregular, ora positivo ora negativo.

Notas explicativas:

${ }^{1}$ A metodologia utilizada pela Fundação João Pinheiro (2013) considera déficit habitacional qualquer domicílio com as seguintes características: habitação precária, coabitação familiar, ônus excessivo de aluguel ou adensamento excessivo de moradores em imóveis alugados.

${ }^{2}$ Esse indicador é realizado pela Federação das Indústrias do Estado de Pernambuco (Fiepe) com o Sindicado da Industria da Construção Civil no Estado de Pernambuco (Sinduscon/ $\mathrm{PE}$ ), o Sindicato da Habitação (Secori/PE) e a Associação das Empresas do Mercado Imobiliário de Pernambuco (ADEMI/PE) e sua amostragem abrange $70 \%$ das ofertas do mercado imobiliário da Região Metropolitana do Recife. Ressalta-se que nessa coleta apenas são considerados os imóveis novos.

\section{REFERÊNCIAS}

ÂNGELA J. Há mercado fora do Recife. NE10, Recife, 31 out. 2013. Disponível em: < http://especiais.jconline.ne10.uol.com.br/construcao_2013/materia_04. php >. Acesso em: 22 nov. 2013.

BOX, G. E. P.; JENKINS, G. M.; REINSEL, G. C. Times Series Analysis, Forecast and Control. 3. ed. Emglewood Cliffs, NJ: Prentice Hall, 1994.

CAIXA ECONÔMICA FEDERAL. Demanda Habitacional no Brasil. Brasília, DF: CAIXA, 2011.

CARDOSO, A. L. at al. Quando um direito vira produto: impactos do Programa Minha Casa Minha Vida na cidade do Rio de Janeiro. In: SIMPÓSIO NACIONAL DE GEOGRAFIA URBANA, 12., 2013, Rio de Janeiro, Anais... Rio de Janeiro, 2013. Disponível em: < http://www.simpurb2013.com.br/wpcontent/uploads/2013/11/1485_gt02.pdf>. Acesso em: 10 set. 2013.

CARDOSO, A. L.; ARAGÃO, T. A. Reestruturação do setor imobiliário e o papel do Programa Minha Casa Minha Vida. In: SEMINÁRIO DA REDE IBERO-AMERICANA DE PESQUISADORES SOBRE GLOBALIZAÇÃO E TERRITÓRIO (RII), 12., 2012, Belo Horizonte. Anais... Belo Horizonte, out. 2012. Disponível em: < http://www.rii.sei.ba.gov.br/anais/g4/restruturacao\%20 do\%20setor\%20imobiliario\%20e\%20o\%20papel\%20do\%20programa $\% 20$ minha\%20casa\%20minha\%20vida.pdf>. Acesso em: 25 out. 2013. 
ENDERS, W. Applied Econometric Time Series. New Yok: Jonh Wiley \& Sons, 1995.

FUNDAÇÃO JOÃO PINHEIRO. Déficit habitacional municipal no Brasil. Belo Horizonte: Centro de Informações, 2013. Disponível em: < http://www.fjp. mg.gov.br/index.php/docman/cei/deficit-habitacional/216-deficit-habitacionalmunicipal-no-brasil-2010/file > . Acesso em: 15 fev. 2015.

INSTITUTO BRASILEIRO DE GEOGRAFIA E ESTATÍSTICA. Comentários das Contas Nacionais, 2013a. Disponível em: <www.ibge.gov.br>. Acesso em: 22 nov. 2013.

ASSOCIAÇÃO DAS EMPRESAS DO MERCADO IMOBILIÁRIO DE PERNAMBUCO. Pesquisa Anual da Indústria da Construção. 2011. Disponível em: <www.ibge.gov.br>. Acesso em: 22 nov. 2013.

ASSOCIAÇÃO DAS EMPRESAS DO MERCADO IMOBILIÁRIO DE PERNAMBUCO. Pesquisa Mensal de Emprego. 2013b. Disponível em: <www.ibge.gov.br>. Acesso em: 22 nov. 2013.

ÍNDICE DE VELOCIDADE DE VENDAS. Unidade de Economia, Estudos e Pesquisas, ano 18, n. 7, set. 2013. Disponível em: <www.ademi-pe.com.br>. Acesso em: 22 nov. 2013.

MILLS, T. C. Times Series Techniques for Economists. Cambridge: Cambridge University Press, 1990.

SHIMIZU, J. Y. Projeção de Impactos econômicos do Programa Minha Casa Minha Vida: Uma abordagem de Equilíbrio Geral Computável. 2010. Dissertação (Mestrado em Economia)-Universidade Federal de Minas Gerais, 2010. Disponível em: < https://cedeplar.ufmg.br/economia/dissertacoes/2010/ Julio_Yukio_Shimizu.pdf>. Acesso em: 25 out. 2013. 


\section{Como citar este artigo:}

BARBOSA, Karla Gomes; FERREIRA IRMÃO, José. Análise dos impactos - gerados pelo Programa Minha Casa Minha Vida no Índice de Velocidade de 乙 Vendas na Região Metropolitana do Recife. RACE, Revista de Administração

\& Contabilidade e Economia. Joaçaba: Ed. Unoesc, v. 14, n. 2, p. 825-842., maio/ ago. 2015. Disponível em: < http://editora.unoesc.edu.br/index/php/race > Acesso em: dia/mês/ano.

Barbosa, K. G., \& Ferreira Irmão, J. (2015). Análise dos impactos gerados pelo Programa Minha Casa Minha Vida no Índice de Velocidade de Vendas na Região

Metropolitana do Recife. RACE, Revista de Administração Contabilidade e Economia.

14(2), 825-842. Recuperado em dia/mês/ano, de http://editora.unoesc.edu.br/ index/php/race 
